\title{
Effect of polymer solution structure on displacement efficiency
}

\author{
Shi Leiting ${ }^{1 *}$, Chen Lei ${ }^{1}$, Ye Zhongbin ${ }^{1}$, Zhou Wei ${ }^{2}$, Zhang Jian², Yang Jie ${ }^{1}$ \\ and Jin Jianbo ${ }^{3}$
}

${ }^{1}$ State Key Laboratory of Oil and Gas Reservoir Geology and Exploitation, Southwest Petroleum University, Chengdu, Sichuan 610500, China

${ }^{2}$ Research Center of CNOOC, Beijing 100027, China

${ }^{3}$ First Oil Production Plant of Henan Branch Corporation of Sinopec, Nanyang, Henan 474780, China

(C) China University of Petroleum (Beijing) and Springer-Verlag Berlin Heidelberg 2012

\begin{abstract}
In this paper, a series of experiments, including atomic force microscope (AFM), environmental scanning electron microscope (ESEM), and core displacement tests were conducted to investigate the effect of polymer solution structure on solution properties and oil displacement efficiency. The results show that in the HPAM solution polymer coils were formed and then aggregated into a loose structure, while the HAP2010 solution formed a strong network structure, which would significantly improve the solution viscosity and flow resistance so as to upgrade the capacity of piston-like oil displacement in highly permeable porous media. Meanwhile, the retention of the HAP2010 solution at pore throats were also enhanced, which could reduce water production during subsequent water flooding and enlarge the swept volume during polymer flooding. Therefore, enhancing the interaction among polymer molecules is an effective way to improve the displacement efficiency of polymer solutions in heavy oil reservoirs with high permeability.
\end{abstract}

Key words: Polymer, solution structure, loosely-aggregated structure, network structure, displacement efficiency

\section{Introduction}

In water solution, partially hydrolyzed polyacrylamide (HPAM) relies on electrostatic interaction between the charged groups on molecular chains to extend the molecular chains as far as possible and to enlarge the hydrodynamic radius of the molecular coils, which can help to increase solution viscosity and thus to improve the mobility control. During polymer flooding, polymer chains would shrink or degrade due to the effects of temperature, pressure, salinity and shearing, etc. (Levitt and Pope, 2008; Seright et al, 2010; Maerker, 1975), resulting in a decrease in the hydrodynamic volume of polymer coils as well as the solution viscosity. Moreover, when the polymer solution flows through porous media, the effect of shear and tension may destroy the configuration of polymer molecules in solution (Shao et al, 2005) and then limit their capacity in an enhanced oil recovery process.

Enhancement of the tolerance to temperature and salinity of polymer solutions and their shearing strength, as well as other properties, mainly depends on either increasing the

*Corresponding author. email: flygoslt@126.com

Received September 16, 2011 length of polymer chains or introducing functional groups into molecular chains, or both. The longer the polymer chain is (like ultra-high molecular weight HPAM) and the more charged groups that there are on the chains, the stronger the total electrostatic repulsion is and the larger the hydrodynamic radius of the polymer coils will be. Meanwhile, the entanglement of polymer chains will increase so as to improve the solution properties (Xu et al, 2011, Zhang et al, 2008). The tolerance to temperature and salinity and the shear strength of the polymer solution can be improved by introducing some functional groups into polymer chains (such as ionic polymer, associating polymer) (Gao et al, 2005; Huang et al, 2006; Molchanov et al, 2007), depending on intermolecular and intramolecular interaction in the polymer solution (electrostatic force, hydrophobic association, etc.). Among such polymers, hydrophobically associating polyacrylamide (HAP) is a typical structural polymer formed by introducing slightly hydrophobic groups into polymer chains. In water solution, the hydrophobic groups on the molecular chains tend to aggregate together. The polymer molecules will associate and form a reversible physical cross-linking network with increased mechanical strength and consequently improve the viscosity, salt tolerance and shear strength of the polymer solution (Zhang et al, 2011; Reichenbach-Klinke et al, 2011). 
In order to analyze the properties of these two types of polymer solutions (HPAM and HAP solutions) thoroughly, a great deal of research has been done to investigate the effect of polymer molecular weight, concentration, temperature, salinity, calcium and magnesium concentration etc., on polymer coils, molecular configuration and solution structure (Dondos, 1989; Yao et al, 2005; Chen et al, 2006). Moreover, several researchers analyzed thoroughly the polymer coil dimensions, aggregation and configuration, and their effect on solution properties (Semenov and Rubinstein, 2002; Caputo, 2004; Kujawa et al, 2004; Sun et al, 2006) and flow characteristics in porous media (Lu et al, 2010). It is necessary to further study the effect of polymer configuration on the solution structure types and the oil displacement efficiency, analyze the relationship between solution structure and displacement efficiency, and to discuss the polymer solution molecular structures suitable for extreme reservoir conditions.

\section{Experimental}

\subsection{Experimental materials}

Partially hydrolyzed polyacrylamide (HPAM), an industrial product, with a relative molecular weight of $1.0 \times 10^{7}$ and a $20 \%$ degree of hydrolysis was provided by the Daqing Petrochemical Corporation. Hydrophobically associating polyacrylamide (HAP2010), with a relative molecular weight of $1.0 \times 10^{7}$ and a $20 \%$ degree of hydrolysis as well as $1.0 \%$ of hydrophobic groups, was experimentally synthesized. The synthesis of HAP2010 followed the well-established literature method (Shi et al, 2010b)

The water used in experiments was synthesized injection water, in which the mass concentrations of $\mathrm{Na}^{+}+\mathrm{K}^{+}, \mathrm{Ca}^{2+}$, $\mathrm{Mg}^{2+}, \mathrm{SO}_{4}{ }^{2-}, \mathrm{HCO}_{3}^{-}$and $\mathrm{Cl}^{-}$were 2,551, 569, 228, 36.6, 190 and $5,470 \mathrm{mg} / \mathrm{L}$, respectively. The total salinity of the injection water was $9,047 \mathrm{mg} / \mathrm{L}$.

The porous media used were sand packs with permeability of about $2,000 \times 10^{-3} \mu^{2}$. The simulated oil used in displacement experiment was prepared by blending kerosene with degassed crude oil. Its viscosity was $70 \mathrm{mPa} \cdot \mathrm{s}$ at $65^{\circ} \mathrm{C}$.

\subsection{Acquisition of microscopic features of polymer solutions}

Ten microliters of polymer solution was deposited on a freshly cleaved mica surface $(2 \mathrm{~cm} \times 2 \mathrm{~cm})$, and stretched out fully as a film. The film was then examined and imaged with a tapping-mode atomic force microscope (TMAFM)
(Nanoscope IIIa atomic force microscope, Digital Instruments Corporation, USA) at atmospheric conditions.

\subsection{Viscosity measurement of polymer solutions}

A Brookfield viscometer (DV-III) was used to measure the viscosity of polymer solutions at a shear rate of $7.34 \mathrm{~s}^{-1}$ at $65^{\circ} \mathrm{C}$.

\subsection{The dynamic light scattering (DLS) experiment of polymer solutions}

A BIC-200SM laser light scattering system (Brookhaven Instruments Corporation, USA) was used to measure the hydrodynamic radius of the polymer coil $\left(D_{\mathrm{h}}\right)$ by DLS.

\subsection{Flow characteristics in porous media}

Linear sand packs $(20 \mathrm{~cm} \times \varnothing 2.0 \mathrm{~cm})$ were used to measure the resistance factor $\left(F_{\mathrm{r}}\right)$ and the residual resistance factor $\left(F_{\text {rr }}\right)$ at $65^{\circ} \mathrm{C}$, which were created by HPAM and HAP2010 solutions. The experimental procedure was similar to that described previously (Shi et al, 2010a), but the injection rate was fixed at $1 \mathrm{~mL} / \mathrm{min}$.

Slice samples were cut from the porous media after water flooding and after subsequent water flooding, respectively. Then an environmental scanning electron microscope (ESEM, FEI Quanta 450) was used to observe the distribution of residual polymers deposited in the porous media. The temperature was maintained at $-5^{\circ} \mathrm{C}$ and the pressure was controlled at $100 \mathrm{~Pa}$ during the whole observation.

\subsection{Displacement experiments}

Displacement tests were conducted with the equipment shown schematically in Fig. 1. Linear sand packs (50 $\mathrm{cm} \times \varnothing 2.5 \mathrm{~cm}$ ) were used to conduct displacement experiments at $65{ }^{\circ} \mathrm{C}$. The experimental procedures are described as follows:

1) The linear sand pack was evacuated and the saturated with brine at a injection rate of $1 \mathrm{~mL} / \mathrm{min}$ until the pressure was stabilized, and then the permeability and porosity were measured.

2) The water-saturated pack was saturated with simulated oil to measure its oil saturation. The injection rates of the simulated oil were $0.1,0.5$, and $1 \mathrm{~mL} / \mathrm{min}$, respectively.

3) Water was injected into the sand pack at a rate of $1 \mathrm{~mL} /$ min until the water cut reached $94 \%$, and then the oil recovery after water flooding was obtained.

4) About 0.3 PV of the polymer solution was then injected

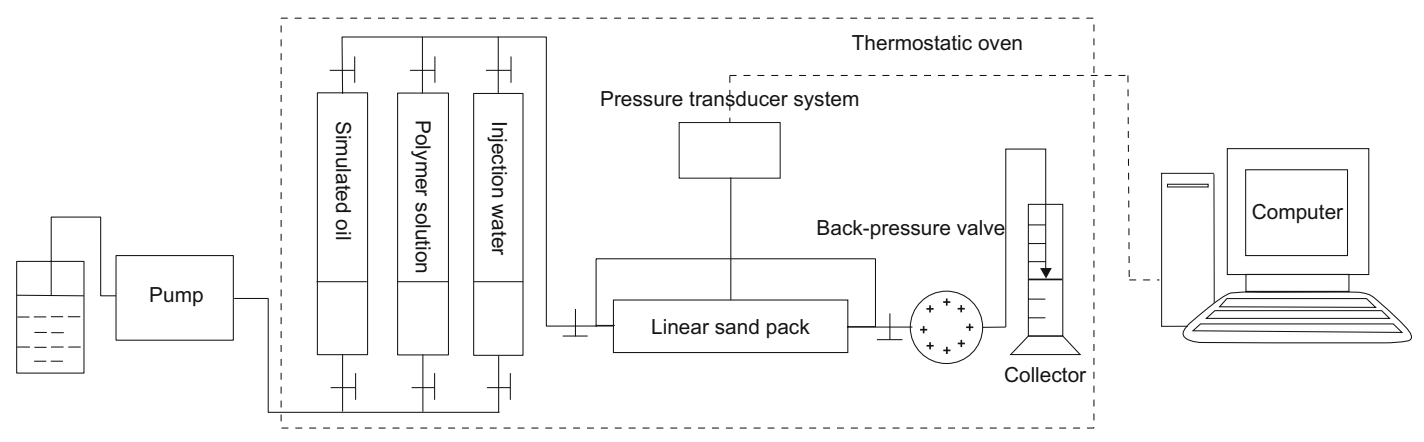

Fig. 1 Schematic of the displacement apparatus 
into the pack, followed by continuous water injection until the water cut reached $96 \%$, and the ultimate oil recovery was obtained.

During displacement experiments, produced oil and water volumes were recorded every five minutes and then the water cut and oil recovery factor were calculated.

\section{Results and discussion}

\subsection{Microscopic features of polymer solutions}

Microscopic characteristics of HPAM and HAP2010 solutions $(1,750 \mathrm{mg} / \mathrm{L})$ were imaged with an atomic force microscope and their microscopic configurations were shown in Fig. 2.

In Fig. 2(a), the molecular coils formed in the HPAM solution were loosely-packed aggregates dispersed with different sizes. While the AFM image of the HAP2010 solution (Fig. 2(b)) shows that in this system an irregular network structure was formed due to inter-molecular interaction. The key factors affecting the distribution of polymer molecules in water solution are the thickness of hydration layer surrounding the polymer chains and the interaction among polymer molecules.

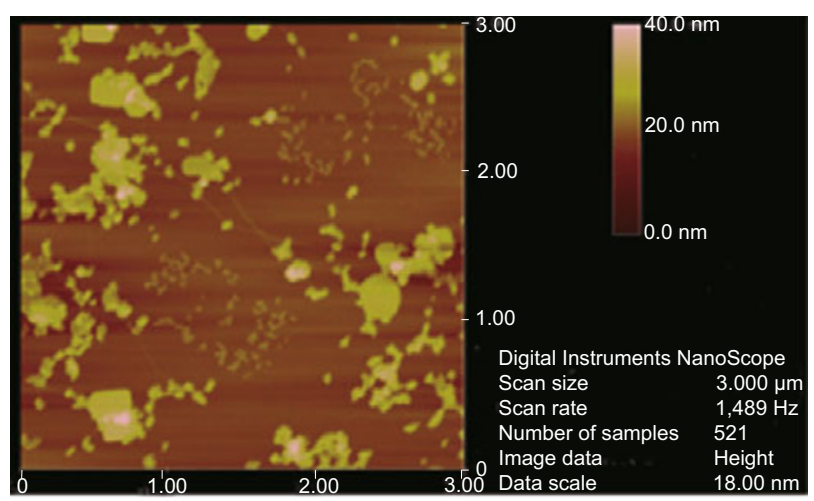

(a) HAPM solution

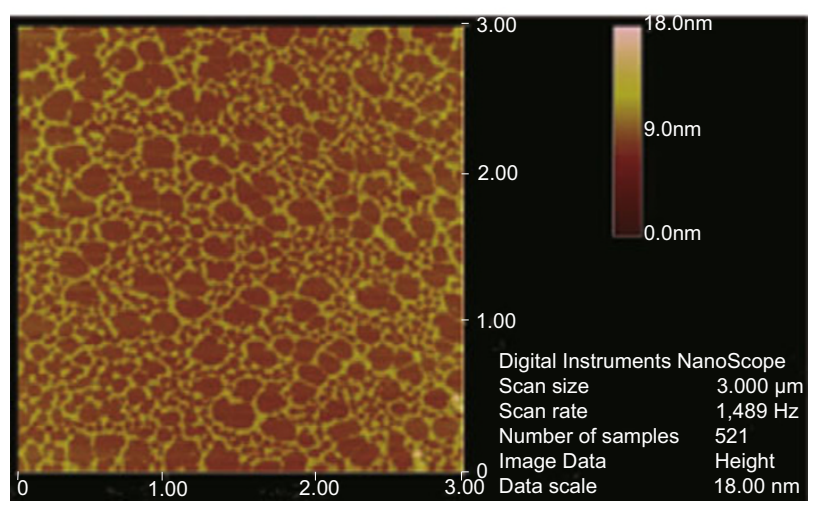

(b) HAP2010 solution

Fig. 2 Observation of polymer solutions by an atomic force microscope

For HPAM, the solution was prepared with highsalinity injection water. Hence, a large number of inorganic cations would enter the electric double layer and combine with carboxylic acid groups with negative charges. This would reduce the electrostatic repulsion between molecular chains, and make the molecular chains curl. Meanwhile, the dehydrating effect of inorganic cations could make the hydrated layer around the molecular chains thinner, so the steric hindrance effect between molecular chains decreased, and the molecular chains shrank (Lu et al, 2010). On the other hand, in high concentration solutions $(1,750 \mathrm{mg} / \mathrm{L})$, polymer chains tended to approach and entangle with each other to form large polymer coils. However, the interaction between polymer coils was too weak to aggregate together easily. The HAP2010 was synthesized by introducing a small number of hydrophobic groups into partially hydrolyzed polyacrylamide, which resulted in hydrophobic association between intermolecular and intramolecular interaction in water solution. Although the shielding effect of inorganic cations in the HAP2010 solution would diminish the electrostatic repulsion and resulted in curling of polymer chains, a large amount of electrolyte existing in the solution could significantly enhance the solution polarity, hence strengthening the association among hydrophobic groups. Polymer chains in the solution of high HAP2010 concentration approached each other and the hydrophobic groups associated and formed strong supramolecular aggregates, and eventually a network structure was formed in the solution.

\subsection{Effects of the solution structure on properties of polymer solutions}

The flow properties of polymer solutions $(1,750 \mathrm{mg} /$ L) were investigated in porous media. The experimental conditions and results were summarized in Table 1 and Fig. 3.

Table 1 Viscosity, resistance factor $F_{\mathrm{r}}$ and residual resistance factor $F_{\mathrm{r}}$ of polymer solutions

\begin{tabular}{ccccc}
\hline $\begin{array}{c}\text { Polymer } \\
\text { solution }\end{array}$ & $\begin{array}{c}\text { Viscosity } \\
\mathrm{mPa} \cdot \mathrm{s}\end{array}$ & $\begin{array}{c}\text { Permeability of the } \\
\text { porous medium } \\
10^{-3} \mu^{2}\end{array}$ & $\begin{array}{c}\text { Resistance } \\
\text { factor }\end{array}$ & $\begin{array}{c}\text { Residual } \\
\text { resistance } \\
\text { factor }\end{array}$ \\
\hline HPAM & 15.7 & 2079 & 23.5 & 2.6 \\
HAP2010 & 298.0 & 2013 & 81.4 & 17.8 \\
\hline
\end{tabular}

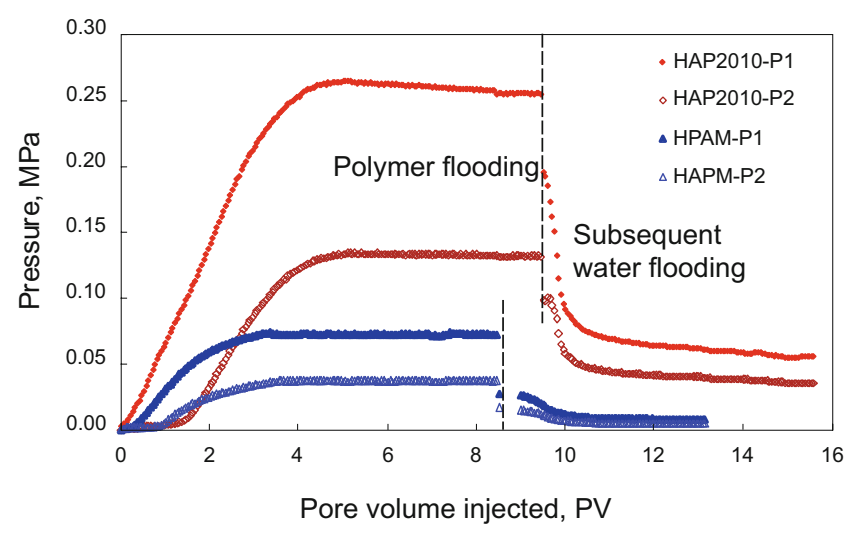

Fig. 3 Relationship between injection pressure and injection pore volume

Fig. 3 shows the pressure curves of polymer solutions flowing through porous media (P1 and P2 present the pressures at the inlet end and half the length of the porous 
media, respectively). It can be seen that hoth the HPAM and HAP2010 solutions had favorable injectivity in porous media.

Table 1 shows that, at the same polymer concentration, the HAP2010 solution had higher viscosity and the ability to build higher resistance to subsequent fluid flow than the HPAM solution. The key factor affecting the viscosity of the polymer solution is the solution structure, which refers to hydrodynamic sizes of the polymers and polymer coils (or aggregates), formed by interaction among polymer chains (Chen et al, 2003). In the HAP2010 solution having a high ion concentration, besides the entanglement among polymer chains, a strong network structure was formed by means of association among aggregates as well and thus to enhance the inner friction. While in the HPAM solution, the increase in viscosity is only due to entanglement and loose packed aggregates of molecular chains, resulting in a limited increase in the viscosity.

The results of the DLS experiment show that the average diameter of polymer coils $\left(D_{\mathrm{h}}\right)$ was $617 \mathrm{~nm}$ for the HAP2010 solution, and $181 \mathrm{~nm}$ for the HPAM solution, respectively. This indicates that the entanglement of polymer chains increased the $D_{\mathrm{h}}$ of the HPAM, yet the molecular chains of HPAM had limited sizes, so the polymer coils dispersed in the solution were only loosely aggregated due to weak attraction, which prevented further increase in polymer coil sizes. Therefore, the HPAM solution had limited viscosity. On the other hand, in the HAP2010 solution, cations made the polymer chains curl and simultaneously enhanced the solution polarity, which improved association of hydrophobic groups, significantly increased the hydrodynamic sizes of polymer aggregates and eventually improved the solution viscosity and flow resistance in the porous media.

The dimension of polymer coils in the HPAM solution was $181 \mathrm{~nm}$. This means that the HPAM solution can easily pass through the porous media. While the HAP2010 solution formed a network structure through association, and a high injection pressure was required to push the solution through the porous media. This means the HAP2010 solution had higher mobility control ability than the HPAM solution.

During subsequent water flooding, the flow resistance created by the HAP 2010 was much higher than that by the HPAM solution, and the residual resistance factors were 2.6 for the HPAM solution and 17.8 for the HAP2010 solution. Meanwhile, the effect of the polymer solution structure on the residual resistance factor was examined by looking at the sand grains in the ESEM after the water and subsequent water flooding had been terminated and the solution had been removed (Figs. 4 and 5).
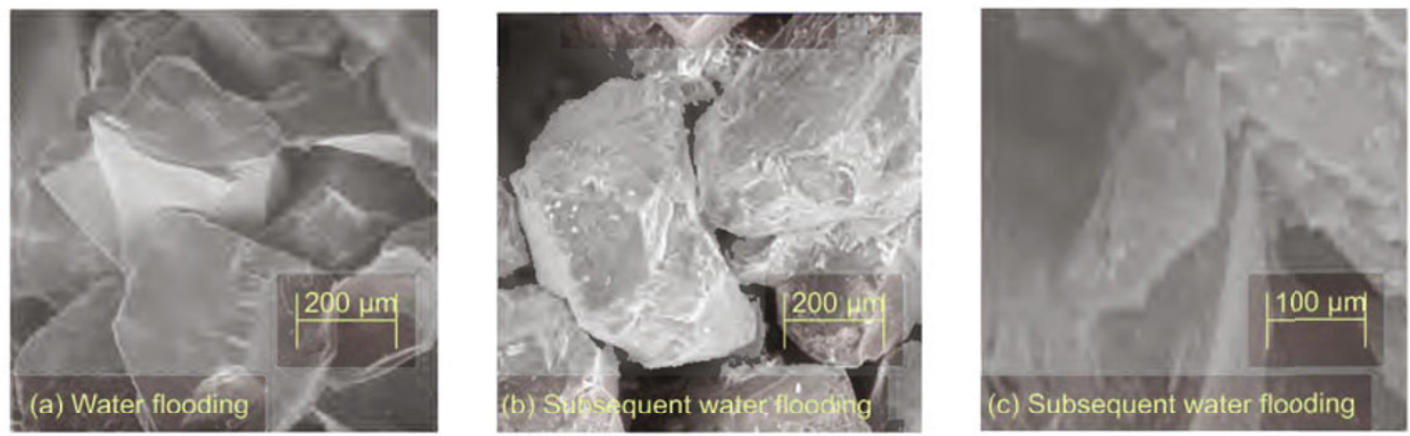

Fig. 4 Images of residual HPAM on sand grains after removal of the solutions
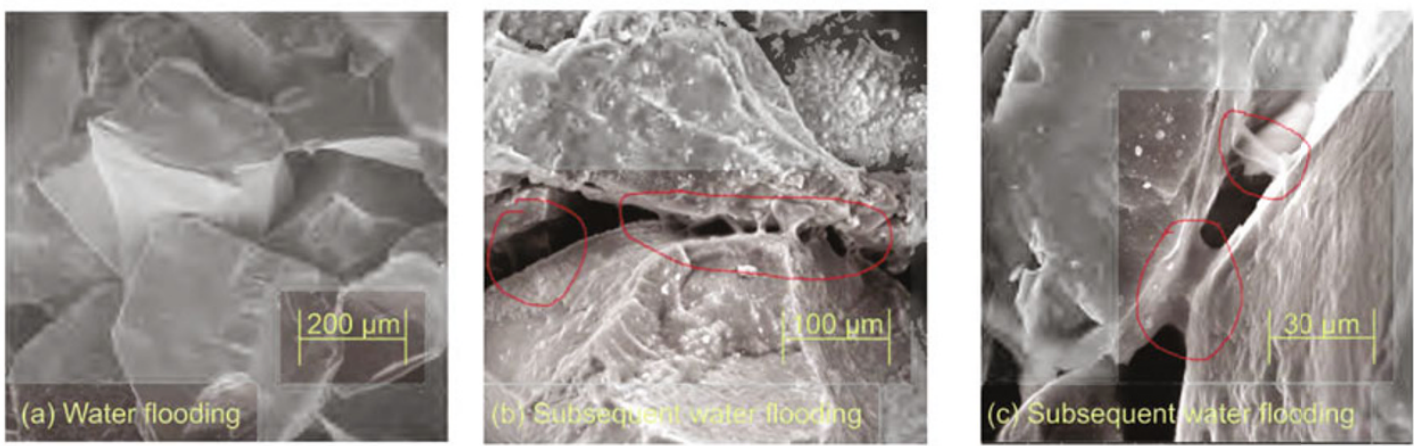

Fig. 5 Images of residual HAP2010 on sand grains after removal of the solutions

In Fig. 4(a), after water flooding, the surface profile of quartz sand grains was very clear and presented some sharp edges and corners. The grain surfaces became smooth after HPAM solution flooding and subsequent water flooding, but there was not a large amount of polymer accumulated in the porous channels (see Fig. 4(b) and (c)), which illustrates that the reduction in permeability of porous media mainly depended on the adsorption of HPAM on rock surfaces ( $Z$ itha, 1998). As shown in Fig. 5, after injection of the HAP2010 solution, not only did the surface of quartz sand grains become smooth, but it also could be observed that there was a large amount of polymer retained in the porous channels (see 
Fig. 5(b) and (c)), which indicates that the synergetic effect of polymer adsorption and retention would greatly modify the water permeability of the porous media (Ren et al, 2006). This can be explained as follows. The polymer coils in the HPAM solution aggregated loosely and exhibited a loosely-packed structure in solution, and the interaction between coils was relatively weak, so that during the subsequent water flooding, the polymer coils were easily flushed out from the porous media, and as a result the polymer coils could not be retained in the porous media. Therefore, the residual resistance factor of the HPAM solution was low. The HAP2010 solution formed a network structure due to intermolecular association. The shearing and extension effect might partially destroy the network structure when the HAP2010 solution flowed through porous media, yet when shearing and extension diminished or disappeared, the HAP2010 molecules could associate with polymer molecules adsorbed on the rock surface (Taylor and Nasr-El-Din, 2007; Volpert et al, 1998) to increase retention of polymer in the porous media, thereby enhancing the interaction between polymer molecules and rock, and effectively decreasing the water permeability of porous media with high permeability. Therefore, the polymer solution with a network structure formed by intermolecular interaction had higher viscosity and mobility control ability, and could improve polymer performance in high salinity water.

\subsection{Evaluation of oil displacement efficiency}

The displacement experiments of HAP2010 and HPAM solutions $(1,750 \mathrm{mg} / \mathrm{L})$ were carried out on oil-saturated sand packs with an original permeability of $2,087 \times 10^{-3}$ and $2,019 \times 10^{-3} \mu \mathrm{m}^{2}$, respectively (see Fig. 6).

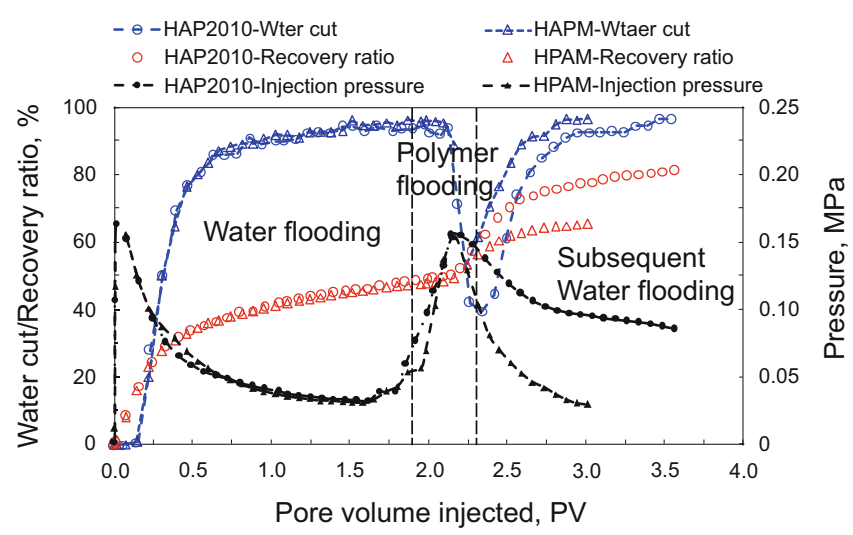

Fig. 6 Water cut, oil recovery factor and pressure curves during flooding

During water flooding, the water cut rose up sharply (see Fig 6) after water breakthrough due to unfavorable oilwater mobility. The ultimate oil recoveries by water flooding were approximately $46 \%$ in both experiments. After polymer flooding and subsequent water flooding, the water cut dropped at first and then rose up rapidly, presenting a "V" shaped trend after the injection of HPAM solution. For HAP2010 flooding, the water cut dropped and remained stable for a period of time, and then rose slowly, showing a "U" shaped trend. In the two displacement experiments, a similar tendency of a gradual increase in the injection pressures was observed during polymer flooding. During subsequent water flooding, the two injection pressures dropped gradually, but the injection pressure of HAP2010 flooding was remarkably higher than that of HPAM flooding. The incremental oil recovery values were $19.4 \%$ for HPAM flooding and $34.2 \%$ for HAP2010.

The injection of the HAP2010 solution into the sand pack was shown to result in a sharp reduction of water cut and to slow down the rate of water cut increase during the subsequent water flooding. The network structure of the HAP2010 solution formed in porous media could create high viscosity and flow resistance, hence effectively enlarged the swept volume and decreased the water cut. Meanwhile, the strong network structure could decrease the viscosity loss due to polymer adsorption and retention along the way, which improved the capacity of piston-like displacement, delayed the displacing front breakthrough (Aktas et al, 2008), and prolonged the low water cut oil production period. Furthermore, the HAP2010 solution could significantly decrease the water phase permeability by means of adsorption and retention in the porous media. Therefore, the HAP2010 solution exhibited excellent performance in increasing the injection pressure and enlarging the swept volume during subsequent water flooding. This effectively hindered the water flow and then improved oil production. However, the molecular coils of HPAM were dispersed in the solution, leading to relatively low viscosity. Moreover, in highpermeability heavy oil reservoirs, it is difficult to improve the water-oil mobility ratio for the water flood by merely enhancing the adsorption of polymers in the porous media, which might limit the ability of HPAM solution to enlarge the swept volume. Besides, due to its smaller $D_{\mathrm{h}}$, the HPAM slug was rapidly broken through and then a water channel was formed during subsequent water flooding, which resulted in a sharp increase in water cut and eventually lowered displacement efficiency. Therefore, the HAP2010 solution with a network structure formed by intermolecular interaction (like association) was more suitable for high permeability heavy oil reservoirs.

\section{Conclusions}

1) A polymer solution, in which polymer coils form a network structure due to intermolecular association, had higher viscosity and mobility control ability than one containing only loosely-aggregated polymer coils.

2) The HPAM solution slug with loosely-aggregated polymer coils was easily broken through and flushed out; while the HAP2010 solution with a network structure could increase the retention of polymer in pore channels, and thus significantly reduce the water permeability of the porous media.

3) The network structure of the polymer solution formed in the porous media could significantly increase flow resistance and cause a sharp drop in water cut and a delay of reaching high water cut $(96 \%)$ in subsequent water flooding. Therefore, this type of polymer solutions had excellent 
mobility control ability and high displacement efficiency.

\section{Acknowledgements}

This paper was supported by the National Science and Technology Major Project (2011ZX05024-004) and National High Technology Research and Development Program of China (863 Program: 2007AA090701-3).

\section{References}

Aktas F, Clemens T, Castanier L, et al. Viscous oil displacement via aqueous associative polymers. Paper SPE 113264 presented at SPE/ DOE Symposium on Improved Oil Recovery, 19-23 April 2008, Tulsa, Oklahoma, USA

Caputo M R, Selb J and Candau F. Effect of temperature on the viscoelastic behaviour of entangled solutions of multisticker associating polyacrylamides. Polymer. 2004. 45(1): 231-240

Chen H, Han L J, Xu P, et al. The thickening mechanism study of hydrophobically modified polyacrylamide. Acta Phys-Chinica Sinica. 2003. 19(11): 1020-1024 (in Chinese)

Chen H, Ye Z B, Han L J, et al. A study on hydrophobically modified polyacrylamide using a fluorescence probe. Chemistry Online. 2006. (1): 26-30 (in Chinese)

Dondos A, Tsitsilianis C and Staikos G. Viscometric study of aggregation phenomena in polymer dilute solutions and determination of the critical concentration c**. Polymer. 1989. 30(9): 1690-1694

Gao B J, Wu N and Li Y B. Interaction between the strong anionic character of strong anions and the hydrophobic association property of hydrophobic blocks in macromolecular chains of a water-soluble copolymer. Journal of Applied Polymer Science. 2005. 96(3): 714722

Huang Z Y, Lu H S and He Y. Amphoteric hydrophobic associative polymer: I. Synthesis, solution properties and effect on solution properties of surfactant. Colloid \& Polymer Science. 2006. 285(3): 365-370

Kujawa P, Audibert-Hayet A, Selb J, et al. Rheological properties of multisticker associative polyelectrolytes in semidilute aqueous solutions. Journal of Polymer Science Part B: Polymer Physics. 2004. 42(9): 1640-1655

Levitt D and Pope G A. Selection and screening of polymers for enhanced-oil recovery. Paper SPE 113845 presented at SPE/DOE Symposium on Improved Oil Recovery, 19-23 April 2008, Tulsa, Oklahoma, USA

Lu X G, Wang X Y, Li Q, et al. The polymer molecular configuration in the oil displacement agent with high temperature and salinity tolerance and its seepage property in the medium-low permeability layer. Acta Chimica Sinica. 2010. 68(12): 1229-1234 (in Chinese)

Maerker J M. Shear degradation of partially hydrolyzed polyacrylamide solutions. SPE Journal. 1975. 15(4): 311-322 (Paper SPE 5101)

Molchanov V S, Philippova O E, Khokhlov A R, et al. Self-assembled networks highly responsive to hydrocarbons. Langmuir. 2007. 23(1): $105-111$
Reichenbach-Klinke R, Langlotz B, Wenzke B, et al. Associative copolymer with favorable properties for the application in polymer flooding. Paper SPE 141107 presented at SPE International Symposium on Oilfield Chemistry, 11-13 April 2011, Texas, USA

Ren K, Wang J L, Lin M Q, et al. Rheological properties of hydrophobically associating polyacrylamide solution. Petroleum Science. 2006. 3(2): 66-72

Semenov A N and Rubinstein M. Dynamics of entangled associating polymers with large aggregates. Macromolecules. 2002. 35(12): 4821-4837

Seright R S, Mozley P S and Han P. Stability of partially hydrolyzed polyacrylamides at elevated temperatures in the absence of divalent cations. Society of Petroleum Engineers. 2010. 15(2): 341-348 (paper SPE 121460)

Shao Z B, Zhou J S, Sun G, et al. Studies of mechanical degradation of partially hydrolized polyacrylamide in course of polymer flooding: Changes in relative molecular mass, viscosity and related parameters. Oilfield Chemistry. 2005. 22(1): $72-77$ (in Chinese)

Shi L T, Ye Z B, Zhang Z, et al. Necessity and feasibility of improving the residual resistance factor of polymer flooding in heavy oil reservoirs. Petroleum Science. 2010a. 7(2): 251-256

Shi L T, Zhang J, Chen H, et al. Preparation and application of a novel polymer flooding agent for EOR. Chinese Patent. CN 201010300045.2. 2010b-08-11

Sun H Q, Zhang K L, Chen J, et al. Effect of hydrophobic association on structure and rheological behavior of polyacrylamide based solutions. Acta Polymerica Sinica. 2006. (6): 810-814 (in Chinese)

Taylor K C and Nasr-El-Din H A. Hydrophobically associating polymers for oil field applications. Paper SPE 2007-016 presented at Canadian International Petroleum Conference, 12-14 June 2007, Calgary, Alberta

Volpert E, Selb J, Candau F, et al. Adsorption of hydrophobically associating polyacrylamides on clay. Langmuir. 1998. 14(7): 18701879

Xu D, Liu C Y and Craig S L. Divergent shear thinning and shear thickening behavior of supramolecular polymer networks in semidilute entangled polymer solutions. Macromolecules. 2011. 44(7): 2343-2353

Yao T Y, Liu Q G, Liu W D, et al. Structural images of partially hydrolyzed polyacrylamide. Acta Petrolei Sinica. 2005. 26(5): 81-84 (in Chinese)

Zhang M G, Zhang L H, Jiang B, et al. Rheological properties of salttolerant HPAM solutions with ultrahigh molecular weight. Journal of Central South University of Technology. 2008. 15(1): 93-97

Zhang P, Wang Y F, Chen W H, et al. Preparation and solution characteristics of a novel hydrophobically associating terpolymer for enhanced oil recovery. Journal of Solution Chemistry. 2011. 40(3): 447-457

Zitha P L J, Van Os K G S and Denys K F J. Adsorption of linear flexible polymers during laminar flow through porous media. Paper SPE 39675 presented at SPE/DOE Improved Oil Recovery Symposium, 19-22 April 1998, Tulsa, Oklahoma

(Edited by Sun Yanhua) 